\title{
Communication
}

\section{Thermal Noise in Cubic Optical Cavities}

\author{
Guanjun $\mathrm{Xu}{ }^{1,2,+}{ }^{-}$, Dongdong Jiao ${ }^{2,+}$, Long Chen ${ }^{2}$, Linbo Zhang ${ }^{2}{ }^{(0)}$, Ruifang Dong ${ }^{2, *}$, Tao Liu ${ }^{2}$ \\ and Junbiao Wang ${ }^{1}$
}

1 School of Mechanical Engineering, Northwestern Polytechnical University, 127 West Youyi Road, Xi'an 710072, China; xuguanjun@ntsc.ac.cn (G.X.); wangjunb@nwpu.edu.cn (J.W.)

2 National Time Service Center, Chinese Academy of Sciences, 3 East Shuyuan Road, Xi'an 710600, China; jiaodd@ntsc.ac.cn (D.J.); chenlong@ntsc.ac.cn (L.C.); linbo@ntsc.ac.cn (L.Z.); taoliu@ntsc.ac.cn (T.L.)

* Correspondence: dongruifang@ntsc.ac.cn

+ These authors contributed equally to this work.

check for updates

Citation: Xu, G.; Jiao, D.; Chen, L.; Zhang, L.; Dong, R.; Liu, T.; Wang, J. Thermal Noise in Cubic Optical Cavities. Photonics 2021, 8, 261. https://doi.org/10.3390/ photonics 8070261

Received: 11 June 2021

Accepted: 5 July 2021

Published: 6 July 2021

Publisher's Note: MDPI stays neutral with regard to jurisdictional claims in published maps and institutional affiliations.

Copyright: (c) 2021 by the authors. Licensee MDPI, Basel, Switzerland. This article is an open access article distributed under the terms and conditions of the Creative Commons Attribution (CC BY) license (https:/ / creativecommons.org/licenses/by/ $4.0 /)$.

\begin{abstract}
Thermal noise in optical cavities sets a fundamental limit to the frequency instability of ultra-stable lasers. Numata et al. derived three equations based on strain energy and the fluctuationdissipation theorem to estimate the thermal noise contributions of the spacer, substrates, and coating. These equations work well for cylindrical cavities. Extending from that, an expression for the thermal noise for a cubic spacer based on the fluctuation-dissipation theorem is derived, and the thermal noise in cubic optical cavities is investigated in detail by theoretical analysis and finite element simulation. The result shows that the thermal noise of the analytic estimate fits well with that of finite element analysis. Meanwhile, the influence of the compressive force $F_{p}$ on the thermal noise in cubic optical cavities is analyzed for the first time. For a $50 \mathrm{~mm}$ long ultra-low expansion cubic cavity with fused silica substrates and GaAs/AlGaAs crystalline coating, the displacement noise contributed from every $F_{p}$ of $100 \mathrm{~N}$ is about three times more than that of the substrate and coating.
\end{abstract}

Keywords: thermal noise; cubic optical cavities; ultra-stable laser; strain energy; finite element analysis

\section{Introduction}

Ultra-stable lasers have many applications, such as frequency metrology [1-5], gravitational wave detection [6], fundamental physics tests [7,8], and coherent optical links [9,10]. A state-of-the-art ultra-stable laser shows that the linewidth is less than $10 \mathrm{mHz}$. The fractional frequency instability reaches a $10^{-17}$ order of magnitude at $1 \mathrm{~s}[11,12]$. Ultra-stable lasers can be achieved by locking the lasers onto optical cavities with the Pound-DreverHall (PDH) technique, and the frequency instability of the ultra-stable laser is then defined by the stability of the optical length of the optical cavity $[13,14]$. The fractional length stability of optical cavities is inevitably limited by thermal noise in the cavity. Several thermal noise sources have been identified, such as Brownian thermal noise and thermo-elastic noise. Among them, the influence of Brownian thermal noise is quite remarkable, and considerable effort has been invested in reducing it [15-24]. Y. Levin proposed an efficient way to calculate thermal noise based on the fluctuation-dissipation theorem (FDT) and some significant efforts have been expended to reduce it [15-24]. A set of equations was given by Numata et al. [16] for estimating the noise contribution of a spacer, substrates, and coating of cylindrical cavities using this approach. High mechanical quality substrates and coating materials are being used in state-of-the-art optical cavities to reduce the thermal noise of the optical cavity [11-14].

The cubic optical cavity is based on a cubic geometry with four supports placed symmetrically about the optical axis in a tetrahedral configuration [25]. It plays an essential role for transportable ultra-stable lasers, which are used outside the laboratory for applications such as space optical clocks, geodesy, tests of fundamental physics in space, and the generation of ultra-stable microwaves for radar [25-41]. A compressive force $F_{p}$ 
must be applied to fix the cavity to resist the extreme vibrations and impact of rocket launching. Previous studies have shown that the cubic optical cavity can still maintain a low vibration sensitivity in a wide compressive range $[25,40]$. However, a large $F_{p}$ will increase the elastic energy of the optical cavity, inducing thermal noise and adversely affecting applications of ultra-stable lasers in some high-precision fields [25-41]. Therefore, to evaluate the performance of transportable ultra-stable lasers using a cubic cavity, it is necessary to propose a reasonable method to analyze the thermal noise in a cubic cavity.

Detailed theoretical analysis and finite element simulation (FEA) of the Brownian thermal noise in cubic optical cavities are presented herein. First, based on FDT and strain energy, the theoretical estimation formula of the Brownian thermal noise in a cubic spacer is derived. We compare the new analysis method and FEA for the same conditions. The two results are in good agreement. The new analysis formula in this work fits well with any edge dimension of the cubic spacer. A complete explanation of the divergence between the simulation and analytical estimation is presented in detail. Second, the influence of $F_{p}$ on the thermal noise in cubic optical cavities is investigated for the first time. For a 50 $\mathrm{mm}$ long ultra-low expansion (ULE) optical cavity, the displacement noise contributed from each $100 \mathrm{~N} F_{p}$ is about three times that of FS substrates and GaAs/AlGaAs crystalline coating when the $F_{p}$ is larger than $100 \mathrm{~N}$.

The paper is organized as follows: In Section 2, the theoretical framework of the thermal noise in the cubic optical cavities is discussed. The FEA simulation is applied to a typical cubic optical cavity design in Section 3.1. The deviations from the analytical estimate for the cubic spacer are discussed. To better understand the divergence between the simulation and analytical estimation, the thermal noise of cubic spacers under various conditions is investigated in Section 3.2, which focuses on the influence of $F_{p}$, the materials, and the edge length of the cubic optical cavity. In Section 4, the thermal noise of cubic optical cavities of mixed materials is investigated. The conclusions are summarized in Section 5.

\section{Theoretical Framework}

The effect of Brownian motion thermal noise on the length stability of optical cavities has been discussed by Numata et al. [16], following Y. Levin's direct approach [15]. For clarity, this concept is illustrated by the following example.

Assuming that $\mathrm{x}$ is the distance between two mirrors of the optical cavity, and based on FDT, the power spectral density $S_{x}(f)$ of the optical length fluctuations along the $x$-axis can be expressed as [16].

$$
S_{x}(f)=\frac{4 k_{B} T U \phi}{\pi f F^{2}}
$$

where $k_{B}$ is the Boltzmann constant, $T$ is the temperature, $F$ and $f$ are the amplitude and frequency of an oscillatory force [16], respectively. $U$ is the maximum elastic strain energy, and $\phi$ is the loss angle of the materials.

For an optical cavity, $S_{x}(f)$ is mainly caused by the spacer, substrate, and coating. The analytic estimates of $S_{x}(f)$ for substrates and coatings in this work are cited in Ref. [16]. Based on Y. Levin's direct approach [15], the analytic estimate of $S_{x}(f)$ for a cubic spacer shown in Figure 1 can be expressed as

$$
\left.\begin{array}{c}
U_{s p a}^{c u b}=\frac{L_{c u b} F^{2}}{2\left(L_{c u b}^{2}-2 l_{c u b}^{2}-\pi r_{s p a}^{2}\right) E_{s p a}} \\
S_{x}(f)=\frac{4 k_{B} T}{\pi f} \frac{L_{c u b}}{2\left(L_{c u b}^{2}-2 l_{c u b}^{2}-\pi r_{s p a}^{2}\right) E_{s p a}} \phi_{s p a}
\end{array}\right\}
$$

where $S_{x}(f)_{s p a}^{c u b}$ is the power spectral density of length fluctuations. $E_{s p a}$ is the elastic modulus of the spacer. This estimate focuses on the cubic spacer. As an estimate for the spacer contribution to the optical cavity noise, the thermal fluctuations of the length of a cube, averaged over the spacer whole front face area, are calculated. Y. Levin's direct approach demands a that is pressure uniformly distributed across its front faces with 
the area. Equation (2) takes into account that the areas of the central bore and the cut vertices reduce the front face area of the spacer. To derive these equations, the formula for the position fluctuations of one end of a free elastic bar is used. According to $Y$. Levin's direct approach, calculating the length fluctuation requires applying opposite forces simultaneously to both ends.
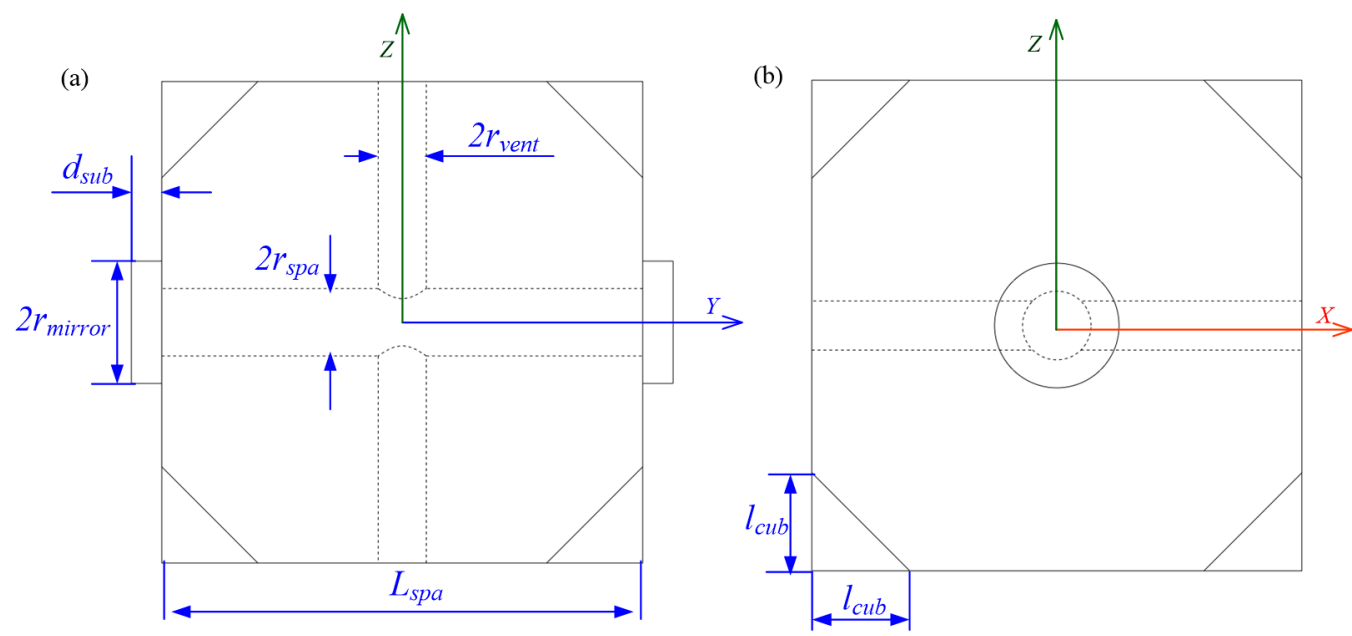

Figure 1. Sketch of the cubic optical cavity model with dimensions used for the analysis and FEA simulations in this publication. (a) Side view of the cubic optical cavity. (b) Front view of the cubic optical cavity.

According to Ref. [16], the power spectral density of the length fluctuations may be converted to power spectral density of the frequency noise, which can be conventionally characterized by the Allan deviation $\sigma_{y}$ of the fractional frequency fluctuations $y$ and expressed as

$$
\sigma_{y}=\frac{\sqrt{2(\ln 2) f S_{v}(f)}}{v}=\frac{\sqrt{2(\ln 2) f S_{x}(f)}}{L}
$$

From Equations (2) and (3), a strain energy of $0.1 \mathrm{~nJ}$ corresponds to a length fluctuation $S_{x}(f)=0.858 \times 10^{-35} \mathrm{~m}^{2} / \mathrm{Hz}$ at $1 \mathrm{~Hz}$ and a corresponding Allan deviation of $\sigma_{y}=6.88 \times 10^{-17}$ at $1 \mathrm{~s}$ for a $50 \mathrm{~mm}$ long ULE cavity [17].

\section{Estimation Calculation and Simulation}

The FEA software is used to calculate the strain energy and thermal noise in the cubic optical spacer. As the thermal noise of the cavity is proportional to strain energy, the results are given in terms of elastic strain energy for convenience. The most important parameters used in the calculation, including dimensions and materials, are given in Tables 1 and 2.

Table 1. Parameters used for estimation and simulation.

\begin{tabular}{ccccccc}
\hline Parameters & $d_{\text {sub }}(\mathrm{mm})$ & $\boldsymbol{r}_{\text {mirror }}(\mathbf{m m})$ & $\boldsymbol{r}_{\text {vent }}(\mathrm{mm})$ & $\boldsymbol{r}_{\text {spa }}(\mathrm{mm})$ & $\boldsymbol{l}_{\text {cub }}(\mathrm{mm})$ & $\boldsymbol{k}_{B}$ \\
\hline Value & 6.3 & 12.7 & 3 & 7 & 16.4 & $1.381 \times 10^{-23} \mathrm{~J} / \mathrm{K}$ \\
\hline
\end{tabular}

Table 2. Material properties.

\begin{tabular}{cccccccc}
\hline Material Properties & ULE [18] & FS [1-8] & $\begin{array}{c}\text { Silica } \\
<\mathbf{1 1 1}>\text { [14] }\end{array}$ & $\begin{array}{c}\text { Zerodur } \\
\text { [21] }\end{array}$ & $\begin{array}{c}\text { Sapphire } \\
\text { [42] }\end{array}$ & $\begin{array}{c}\mathrm{SiO}_{2} / \mathrm{Ta}_{2} \mathbf{O}_{5} \\
\text { [21] }\end{array}$ & $\begin{array}{c}\mathbf{G a A s} / \mathrm{Al} \\
\mathbf{G a A s}[21]\end{array}$ \\
\hline Elastic modulus (GPa) & 67.6 & 73 & 187.5 & 91 & 400 & 91 & 100 \\
Poisson ratio & 0.17 & 0.16 & 0.23 & 0.24 & 0.29 & 0.19 & 0.32 \\
Loss angle & $1.67 \times 10^{-5}$ & $10^{-6}$ & $10^{-7}$ & $3 \times 10^{-4}$ & $3 \times 10^{-9}$ & $4 \times 10^{-4}$ & $2.5 \times 10^{-5}$ \\
\hline
\end{tabular}


To increase the simulation accuracy, approximately 200,000 tetrahedral elements were used. We assumed that the pressure distribution on the mirror was in accordance with the laser beam profile, and a Gaussian laser beam with a $1 / e^{2}$ beam radius $\omega$ of $250 \mu \mathrm{m}$ was used. Thus, the pressure distribution was [17]:

$$
p(r)= \pm \frac{2 F}{\pi \omega^{2}} e^{-\frac{2 r^{2}}{\omega^{2}}}
$$

According to FDT, $F$ is the force applied at the location where the laser beam hits the mirrors. The absolute value of the force is not critical, provided the stress-strain relationship of the elastic-mechanical system remains linear. For simplicity, in the calculation, we have used a force $F$ of $1 \mathrm{~N}$.

\subsection{Comparison of Estimation and Simulation}

As shown in Figure 2, most of the elastic strain energy for the cubic optical cavity is concentrated in the mirror substrate, similar to the cylindrical and spindle cavity case $[16,17,23]$. The deformation shape of the substrate reflects the Gaussian beam profile. For illustration purposes, a beam waist of $2 \mathrm{~mm}$ has been chosen. For the cubic spacer, the maximum elastic strain energy occurs around the central bore near the contact area with the mirrors. Strong local deformations occur at the boundary between substrate and spacer extending into the spacer. At a distance exceeding this critical depth, a homogeneous energy density distribution is obtained. According to Equation (2) in Ref. [16], the analytic estimate of the spacer contribution does not hold for the full spacer length as it neglects local deformations arising from the non-uniform pressure distribution on its front face.

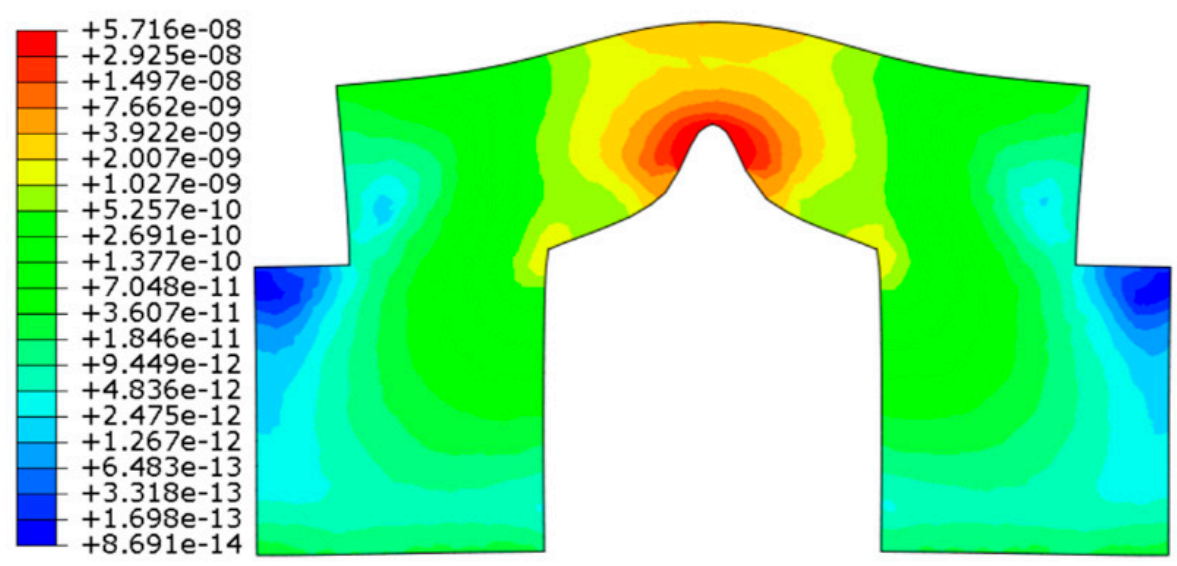

Figure 2. Deformation and contour plot of elastic strain energy density of element (ESEDEN) in the cavity for a Gaussian pressure profile with a $2 \mathrm{~mm}$ waist on the mirror surface. The color coding corresponds to the $\log (\mathrm{ESEDEN})$. The edge $L_{c u b}$ of the cubic optical cavity is $32 \mathrm{~mm}$. Note that the deformation has been amplified by a factor of $9 \times 10^{5}$ for demonstration.

The strain energy for six kinds of cubic optical cavities was estimated and simulated to verify the accuracy of Equation (2). The results are shown in Table 3. For convenience, the length of the cubic optical cavity was chosen according to Ref. [25], which is the cubic optical cavity designed by S. Webster et al. [25]. Furthermore, the other cavity parameters are obtained by calculating the zero vibration sensitivity of the cavity. 
Table 3. The elastic strain energy of the spacer calculated by FEA and two equations.

\begin{tabular}{|c|c|c|c|c|}
\hline \multicolumn{2}{|c|}{ Material } & \multicolumn{3}{|c|}{ Elastic Strain Energy of Spacer (nJ) } \\
\hline \multirow{2}{*}{ Spacer } & \multirow{2}{*}{ Substrate } & \multicolumn{2}{|c|}{ Estimation } & \multirow{2}{*}{ FEA } \\
\hline & & Equation (2) & Reference [16] & \\
\hline ULE & ULE & 0.18 & 0.06 & 0.94 \\
\hline ULE & ULE & 0.18 & 0.06 & 0.93 \\
\hline Zerodur & Zerodur & 0.14 & 0.04 & 0.69 \\
\hline Zerodur & Zerodur & 0.14 & 0.04 & 0.74 \\
\hline Silica & Silica & 0.07 & 0.02 & 0.34 \\
\hline Sapphire & Sapphire & 0.03 & 0.01 & 0.16 \\
\hline
\end{tabular}

As shown in Table 3, the strain energy of FEA is closer to the result calculated by Equation (2) in this paper, differing by a factor of approximately 5 . In comparison, Equation (2) in Ref. [16] differs by a factor of approximately 16. For a pure ULE cubic cavity, compared to the result simulated by FEA, the strain energy of approximately $0.88 \mathrm{~nJ}$ stored in the spacer is underestimated by Equation (2) in Ref. [16]. This underestimation is attributed to the complex local deformations produced by the non-uniform pressure and strain caused in different materials. Kessler et al. [17] and G. Xu et al. [23] have noticed a similar divergence in the case of a cylindrical cavity and spindle cavities. There are two possible reasons for this. One reason is that the transition produces the stress concentration from the mirror to the spacer. The other one is that bending strain energy was not considered. Note that Teflon or Viton pads have not been included in our numerical simulations. T. Kessler et al. [17] studied the contribution of Viton to the thermal noise, and the results showed that the contribution of Viton can be ignored for the widely used optical cavity at present. However, once low-loss coatings with sufficient reflectivities are available, this might need attention.

\subsection{The Effect of Cavity Dimensions on Elastic Energy}

To better understand the divergence between the simulation and analytical estimation, we investigated the thermal noise of cubic spacers under varying conditions, including the spacer's edge length, materials, and $F_{p}$.

\subsubsection{Room Temperature Materials}

As shown in Figure 3, for a cubic optical cavity made of ULE or Zerodur, the strain energy calculated by FEA, the two equations expressed by Equation (2) in this paper, and Equation (2) in Ref. [16] decrease with the increase in cavity length. As Figure 3a,b shows, for the two spacers, the results of FEA are approximately a factor of 5 larger than that of Equation (2) in this paper and a factor of 14 larger than that of Equation (2) in Ref. [16], respectively. For the two spacers, the gaps of strain energy between FEA and Equation (2) in this paper are near-constant offsets of approximately $0.74 \mathrm{~nJ}$ and $0.55 \mathrm{~nJ}$, respectively, and those between the simulation and Equation (2) in Ref. [16] are approximately $0.82 \mathrm{~nJ}$ and $0.6 \mathrm{~nJ}$, respectively. The above results indicate that Equation (2) from this paper is more consistent with the results of FEA than that of Equation (2) in Ref. [16]. 

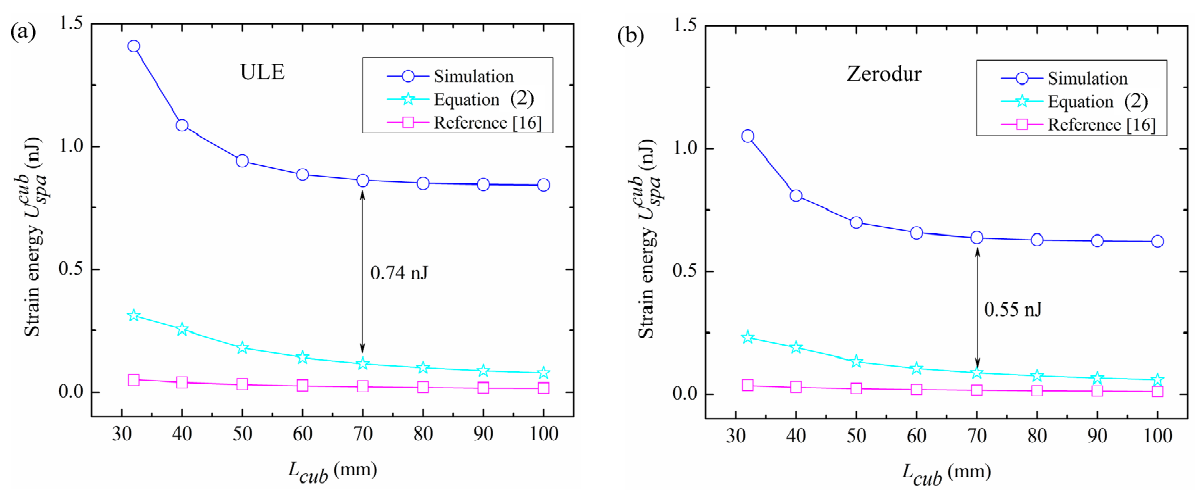

Figure 3. Strain energy as a function of edge $L_{c u b}$ of the cubic spacer. The solid blue line with circles represents the simulation results. Magenta solid line with squares presents the analytic estimate according to Equation (2) in Ref. [16]. Cyan solid line with stars presents the analytic estimate according to Equation (2). (a) ULE cubic spacer with ULE substrates. (b) Zerodur cubic spacer with Zerodur substrates.

\subsubsection{Low-Temperature Materials}

For low-temperature material cubic optical cavities, such as silica and sapphire, Figure 4 shows that the strain energy calculated by Equation (2) in this paper is more consistent with the results of FEA than that of Equation (2) from Ref. [16], which is similar to the case of room temperature materials. For the two spacers, the results of FEA are approximately a factor of 5 greater than that of Equation (2) in this paper and a factor of 18 greater than that of Equation (2) in Ref. [16]. As the spacer edge length increases, for the all-silica and all-sapphire cubic optical cavities, the gap of the strain energy in the spacers between FEA and Equation (2) tends to near-constant offsets of approximately $0.27 \mathrm{~nJ}$ and $0.12 \mathrm{~nJ}$, respectively. We believe that this divergence is similar to the case of room temperature material cubic optical cavities.
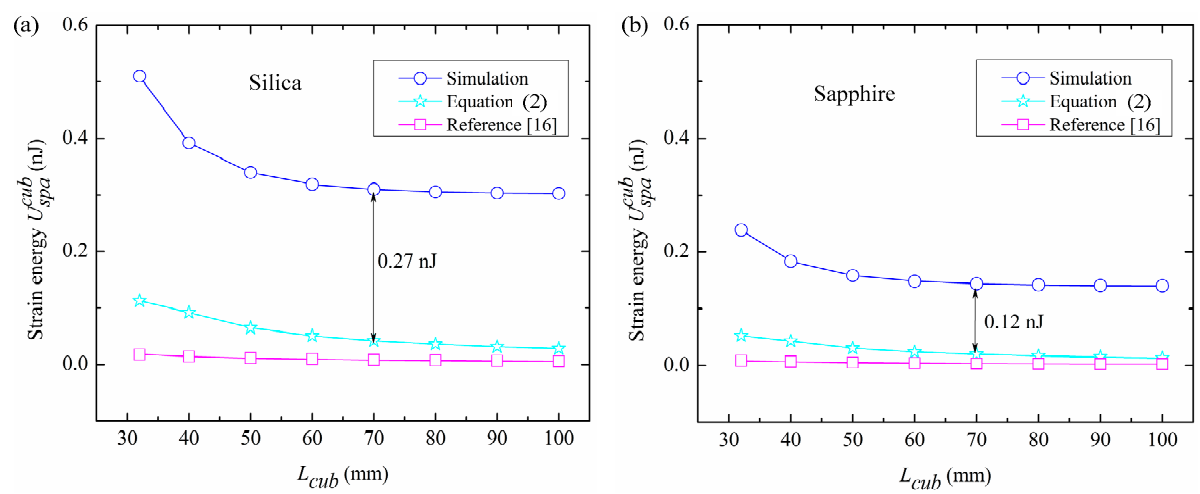

Figure 4. Strain energy as a function of edge $L_{c u b}$ of the cubic spacer. The solid blue line with circles presents the simulation results. Magenta solid line with squares presents the analytic estimate according to Equation (2) in Ref. [16]. Cyan solid line with stars presents the analytic estimate according to Equation (2). (a) Silica cubic spacer with silica substrates. (b) Sapphire cubic spacer with sapphire substrates.

\subsection{The Effect of Compressive Force on Elastic Energy}

To rigidly mount the cubic optical cavity, four $F_{p}$ s were used to load the cut faces of the cubic cavity. $F_{p}$ will change the stress field inside the cubic optical cavity, affecting the strain energy contributed from the force $F$ in Equation (1). Assuming that the strain energy produced by four $F_{p} s$ where $F=0 \mathrm{~N}$ is defined as $U_{p}$, and the strain energy produced by 
four $F_{p} \mathrm{~s}$ and $F=1 \mathrm{~N}$ is $U_{p+F}$, then the strain energy contributed from the force $F=1 \mathrm{~N}$ can be expressed as

$$
U_{F}=U_{p+F}-U_{p}
$$

As shown in Figure $5 b$, the strain energy $U_{F}$ in the spacer of a $50 \mathrm{~mm}$ long ULE cavity as a function of $F_{p}$ is obtained by FEA. The divergence between $U_{F}$ and $U_{0}$ becomes more pronounced as $F_{p}$ increases. Generally, the results show that there is an approximately linear relationship between the strain energy $U_{F}$ and $F_{p}$. When $F_{p}$ is about $80 \mathrm{~N}$, strain energy $U_{F}$ is $1 \mathrm{~nJ}$ larger than $U_{0}$. The strain energy divergences are about $1.2 \mathrm{~nJ}$ and $6.5 \mathrm{~nJ}$, where $F_{p}$ is $100 \mathrm{~N}$ and $500 \mathrm{~N}$. A strain energy of $1.2 \mathrm{~nJ}$ corresponds to an Allan deviation of $\sigma_{y}=2.4 \times 10^{-16}$ at $1 \mathrm{~s}$ for a $50 \mathrm{~mm}$ long ULE cavity. Therefore, an appropriate $F_{p}$ should be chosen for a lower thermal noise of the cubic optical cavity.
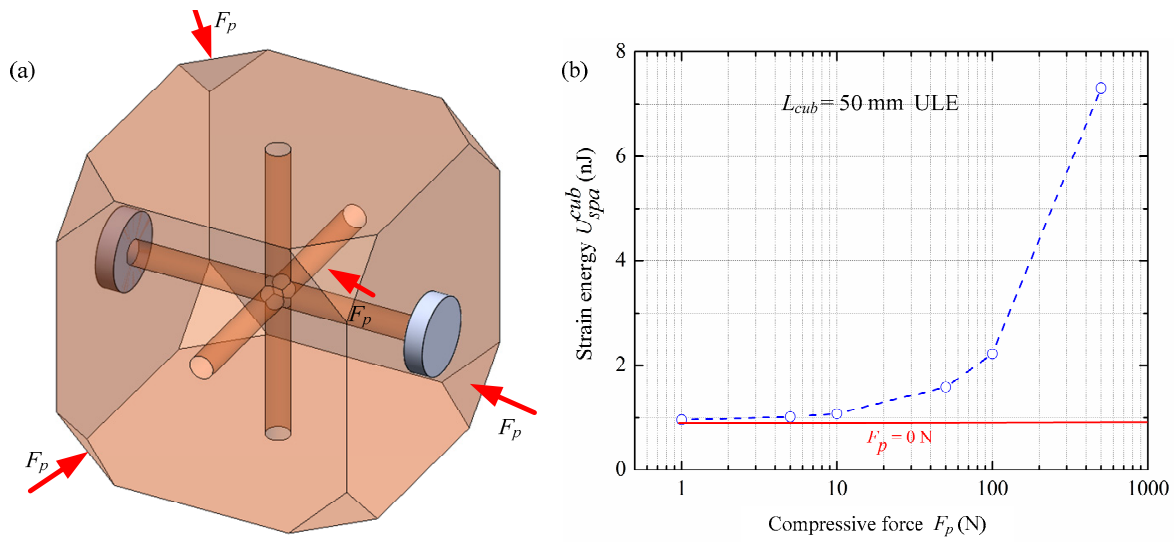

Figure 5. Compressive force model of a cubic optical cavity and strain energy as a function of the compressive force. $F_{p}$ is the compressive force. The red arrows indicate the directions of $F_{p}$. Blue dashed line with circles represents the simulation results of strain energy of cubic optical cavities. When $F_{p}$ is $0 \mathrm{~N}$, the strain energy $U_{0}$ is indicated by a red solid line. Note that $L_{c u b}$ and material of the cubic cavity are $50 \mathrm{~mm}$ and ULE, respectively. (a) Compressive force model of a cubic optical cavity. (b) Strain energy as a function of $F_{p}$.

\section{Mixed Material Cavities}

In cavities with ULE, the major contribution to thermal noise arises from the mirror substrates and coating. Substrate materials with a higher mechanical Q-factor, such as fused silica (FS), have been used to reduce the thermal noise of mirror substrates [17,19]. However, fused silica shows a relatively large room temperature coefficient of thermal expansion (CTE). The large CTE difference in such a mixed material cavity leads to an unwanted lowering of the zero-crossing temperature of the cavity's CTE. Either special mirror configurations have been applied, or additional ULE rings have been optically contacted with the back surfaces of the FS mirrors to compensate for this effect [18].

T. Kessler et al. [17] investigated the thermal noise of a mixed material cavity with a $\mathrm{SiO}_{2} / \mathrm{Ta}_{2} \mathrm{O}_{5}$ coating. The results show that the contribution from the coating to the total thermal noise of the cylindrical cavity is considerable. A GaAs/AlGaAs crystalline coating with a low loss angle was applied to reduce the thermal noise of the coating [21]. This research used the FEA model discussed above to investigate the percent contribution of $S_{x}(f)$ in the mixed material cavity, including the ULE cubic spacer and the FS substrates with a GaAs/AlGaAs crystalline coating and a $\mathrm{SiO}_{2} / \mathrm{Ta}_{2} \mathrm{O}_{5}$ coating. A $50 \mathrm{~mm}$ edge cubic optical cavity was simulated. Additional $6 \mathrm{~mm}$ thick ULE rings with a diameter of $25.4 \mathrm{~mm}$ and a central bore of $14 \mathrm{~mm}$ were added, where $F_{p}$ of $100 \mathrm{~N}$ was applied.

Figure 6 shows the FEA results of the $S_{x}(f)$ contributions caused by the different cavity components. We find that the additional $S_{x}(f)$ of the ULE ring is approximately equal to that of the spacer. Neither can be neglected. Using a GaAs/AlGaAs crystalline coating, the $S_{x}(f)$ contribution in a spacer for an optical cavity with and without ULE rings is about $59 \%$ and 
$32 \%$, respectively, corresponding to the contribution of thermal noise in a spacer to the total thermal noise. The ULE ring noise contributions are much higher than that of the coating thermal noise. However, when using a $\mathrm{SiO}_{2} / \mathrm{Ta}_{2} \mathrm{O}_{5}$ coating, the main contribution of $S_{x}(f)$ in the optical cavity comes from the spacer and coating, and $S_{x}(f)$ in the spacer accounts for $38 \%$ of the total $S_{x}(f)$ for the optical cavity without a ULE ring, corresponding to the proportion of spacer thermal noise in total thermal noise. We have the paradoxical situation that a mixed material cavity with additional rings shows more thermal noise than one without these rings, which is different from a cylindrical optical cavity. The main reason may be attributed to $F_{p}$ resulting in a change in the internal stress in the optical cavity.
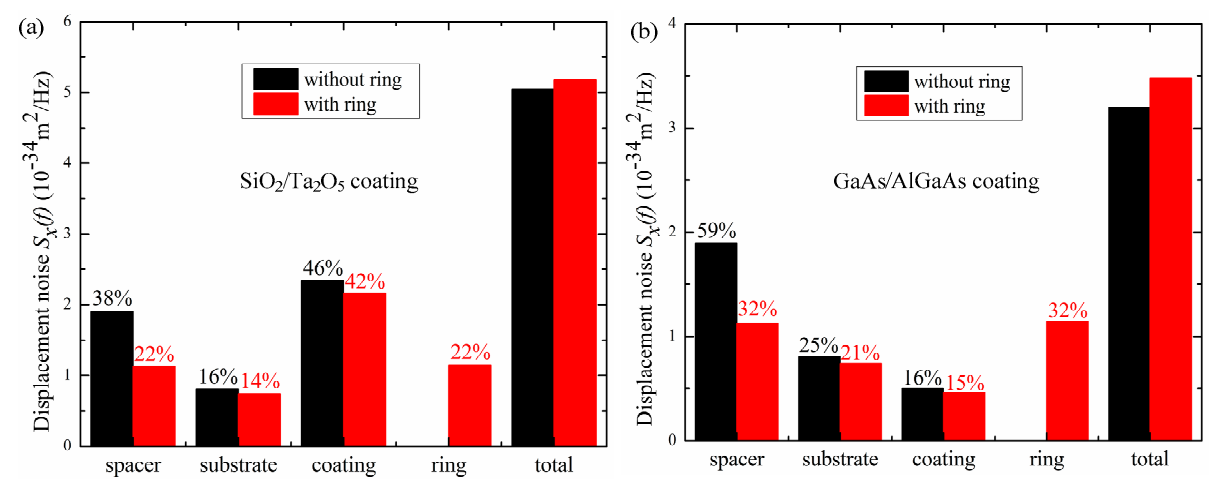

Figure 6. Contributions to the thermal noise of the optical length $S_{x}(f)$ of a cubic optical cavity with and without thermal expansion compensation rings. (a) ULE spacer, FS substrates, $\mathrm{SiO}_{2} / \mathrm{Ta}_{2} \mathrm{O}_{5}$ coating, and ULE rings, the thickness $d_{c o a}=2 \mu \mathrm{m}$ cited from Refs. [17,43]. (b) ULE spacer, FS substrates, GaAs / AlGaAs crystalline coating, and ULE rings, the thickness $d_{c o a}=6.83 \mu \mathrm{m}$ cited from Ref. [21].

In mixed material cavities with FS substrates and an AlGaAs crystalline coating, the ULE spacer limits performance of the cubic optical cavity. Contributions to the thermal noise from the optical length $S_{x}(f)$ as functions of the spacer length $L_{c u b}$ and $F_{p}$ are shown in Figure 7. Under the condition of $F_{p}=0 \mathrm{~N}$, when the spacer length $L_{c u b}$ is greater than $60 \mathrm{~mm}$, the contribution of $S_{x}(f)$ from the spacer equals that of the substrates. The total $S_{x}(f)$ in the cubic optical cavity tends to a constant. As shown in Figure $7 \mathrm{~b}, S_{x}(f)$ in the cubic cavity is proportional to $F_{p}$. For a $50 \mathrm{~mm}$ long ULE cubic optical cavity, when $F_{p}$ is greater than $100 \mathrm{~N}$, the displacement noise contributed from every $100 \mathrm{~N} F_{p}$ is about three times that of FS substrates and the GaAs/AlGaAs crystalline coating, corresponding to the contribution to the thermal noise by $F_{p}$.
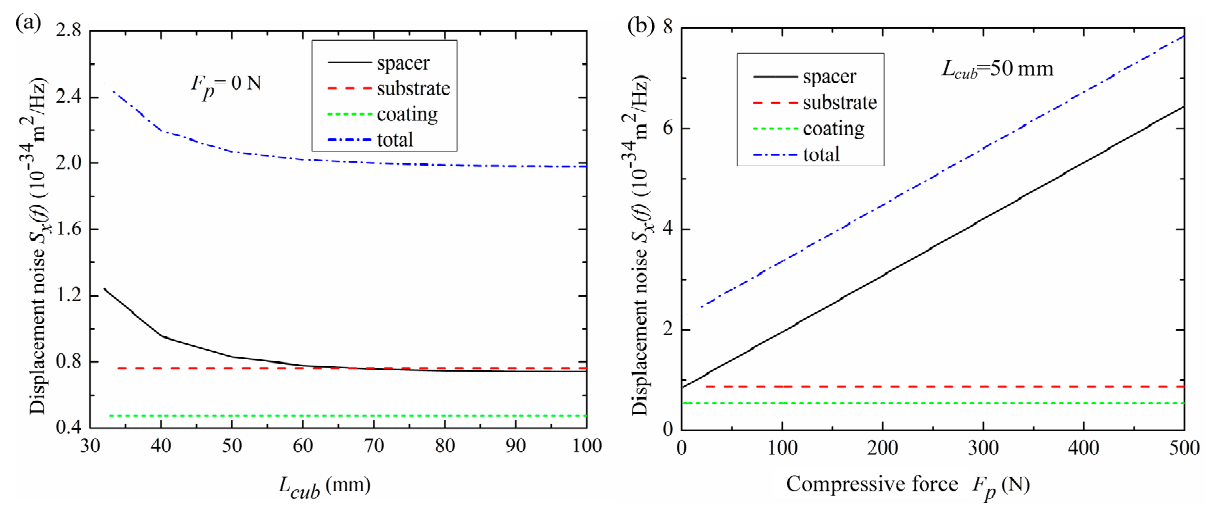

Figure 7. Contributions to the thermal noise of the optical length $S_{x}(f)$ as functions of the spacer length $L_{c u b}$ and $F_{p}$. The spacer is made of ULE. The substrates and coating of the two mirrors are FS and GaAs/AlGaAs crystalline, respectively. (a) The spacer length $L_{c u b}$. (b) Compressive force $F_{p}$. 


\section{Discussions and Conclusions}

This research investigates the thermal noise in cubic optical cavities in detail through theoretical analysis and FEA. We derive an analytic formula for the thermal noise in the cubic spacer based on the FDT theory and strain theory. The calculation results based on this formula demonstrate qualitative agreement with that of the simulation. Compared with the previous estimation formula, this formula is more suitable for the estimation of the thermal noise of the cubic optical cavity. The reasons for the divergence of the results between the theoretical analysis and FEA are also provided.

For the cubic optical cavity, a compressive force $F_{p}$ must be applied. We investigate the influence of the compressive force $F_{p}$ on the thermal noise in cubic optical cavities in detail. This contribution to thermal noise has not been previously considered. To our knowledge, this is the first study of the compressive force influence $F_{p}$ by numerical simulations.

For a $50 \mathrm{~mm}$ long mixed material cubic cavity with a ULE spacer and FS substrates, the displacement noise contributed from every $100 \mathrm{~N}$ of $F_{p}$ is about three times that of the FS substrates and the GaAs/AlGaAs crystalline coating when $F_{p}$ is larger than $100 \mathrm{~N}$. The results show that $F_{p}$ is the dominant noise source for some material choices and cavity geometries. Future studies about the thermal noise contributions in other shapes of optical cavities with different geometries should focus on the compressive force $F_{p}$, such as the spherical optical cavities. The results of this research offer guidance for cubic optical cavity design and minimizing the thermal noise.

Author Contributions: Conceptualization, G.X. and D.J.; methodology, G.X.; software, D.J.; validation, G.X. and D.J.; investigation, G.X.; data curation, L.Z.; writing-original draft preparation, G.X.; writing-review and editing, G.X.; visualization, L.C. and L.Z.; supervision, R.D.; project administration, T.L. and J.W. All authors have read and agreed to the published version of the manuscript.

Funding: The project is partially supported by the Youth Innovation Promotion Association of the Chinese Academy of Sciences (Grant No. 1188000XGJ), the Chinese National Natural Science Foundation (Grant No. 11903041), and the Young Innovative Talents of the National Time Service Center of the Chinese Academy of Sciences (Grant No. Y917SC1).

Acknowledgments: We would like to acknowledge contributions of the strain energy theory of the optical cavity to this paper from X. Zhang of Northwestern Polytechnical University, and F. Sun of National Time Service Center.

Conflicts of Interest: The authors declare no conflict of interest.

\section{References}

1. Katori, H. Optical lattice clocks and quantum metrology. Nat. Photonics 2011, 5, 203-210. [CrossRef]

2. Swallows, M.D.; Bishof, M.; Lin, Y.; Blatt, S.; Martin, M.J.; Rey, A.M.; Ye, J. Suppression of collisional shifts in a strongly interacting lattice clock. Science 2011, 331, 1043-1046. [CrossRef] [PubMed]

3. Huntemann, N.; Okhapkin, M.; Lipphardt, B.; Weyers, S.; Tamm, C.; Peik, E. High-accuracy optical clock based on the octupole transition in 171Yb+. Phys. Rev. Lett. 2012, 108, 090801. [CrossRef]

4. Sherman, J.; Lemke, N.; Hinkley, N.; Pizzocaro, M.; Fox, R.; Ludlow, A.; Oates, C. High-accuracy measurement of atomic polarizability in an optical lattice clock. Phys. Rev. Lett. 2012, 108, 153002. [CrossRef]

5. McFerran, J.J.; Yi, L.; Mejri, S.; Di Manno, S.; Zhang, W.; Guéna, J.; Le Coq, Y.; Bize, S. Neutral atom frequency reference in the deep ultraviolet with fractional uncertainty $=5.7 \times 10^{-15}$. Phys. Rev. Lett. 2012, 108, 183004. [CrossRef]

6. Herrmann, S.; Senger, A.; Mohle, K.; Nagel, M.; Kovalchuk, E.; Peters, A. Rotating optical cavity experiment testing Lorentz invariance at the 10-17 level. Phys. Rev. D 2009, 80, 105011. [CrossRef]

7. Kennedy, C.; Oelker, E.; Robinson, J.M.; Bothwell, T.; Kedar, D.; Milner, W.; Marti, E.; Derevianko, A.; Ye, J. Precision metrology meets cosmology: Improved constraints on ultralight dark matter from atom-cavity frequency comparisons. Phys. Rev. Lett. 2020, 125, 201302. [CrossRef] [PubMed]

8. Chou, C.W.; Hume, D.B.; Rosenband, T.; Wineland, D.J. Optical clocks and relativity. Science 2010, 329, 1630-1633. [CrossRef] [PubMed]

9. Jiang, H.; Kéfélian, F.; Crane, S.; Lopez, O.; Lours, M.; Millo, J.; Holleville, D.; Lemonde, P.; Chardonnet, C.; AmyKlein, A.; et al. Long-distance frequency transfer over an urban fiber link using optical phase stabilization. J. Opt. Soc. Am. B 2008, 25, 2029-2035. [CrossRef] 
10. Predehl, K.; Grosche, G.; Raupach, S.M.F.; Droste, S.; Terra, O.; Alnis, J.; Legero, T.; Hnsch, T.W.; Udem, T.; Holzwarth, R.; et al. A 920-kilometer optical fiber link for frequency metrology at the 19th decimal place. Science 2012, 336, 441-444. [CrossRef] [PubMed]

11. Matei, D.G.; Legero, T.; Häfner, S.; Grebing, C.; Weyrich, R.; Zhang, W.; Sonderhouse, L.; Robinson, J.M.; Ye, J.; Riehle, F.; et al. 1.5 $\mu \mathrm{m}$ Lasers with Sub-10 mHz Linewidth. Phys. Rev. Lett. 2017, 118, 263202. [CrossRef] [PubMed]

12. Robinson, J.M.; Oelker, E.; Milner, W.R.; Zhang, W.; Legero, T.; Matei, D.G.; Riehle, F.; Sterr, U.; Ye, J. Crystalline optical cavity at 4 $\mathrm{K}$ with thermal-noise limited instability and ultralow drift. Optica 2019, 6, 240-243. [CrossRef]

13. Barger, R.L.; Sorem, M.S.; Hall, L.J. Frequency stabilization of a cw dye laser. Appl. Phys. Lett. 1973, 22, 573-575. [CrossRef]

14. Drever, R.W.P.; Hall, J.L.; Kowalski, F.V.; Hough, J.; Ford, G.M.; Munley, A.J.; Ward, H. Laser phase and frequency stabilization using an optical resonator. Appl. Phys. B 1983, 22, 97-105. [CrossRef]

15. Levin, Y. Internal thermal noise in the LIGO test masses: A direct approach. Phys. Rev. D 1998, 57, 110403. [CrossRef]

16. Numata, K.; Kemery, A.; Camp, J. Thermal-noise limit in the frequency stabilization of lasers with rigid cavities. Phys. Rev. Lett. 2004, 93, 250602. [CrossRef] [PubMed]

17. Kessler, T.; Legero, T.; Sterr, U. Thermal noise in optical cavities revisited. J. Opt. Soc. Am. B 2012, 29, 178. [CrossRef]

18. Legero, T.; Kessler, T.; Sterr, U. Tuning the thermal expansion properties of optical reference cavities with fused silica mirrors. J. Opt. Soc. Am. B 2010, 27, 914. [CrossRef]

19. Webster, S.A.; Gill, P. Low-thermal-noise optical cavity. In Proceedings of the 2010 IEEE International Frequency Control Symposium, Newport Beach, CA, USA, 1-4 June 2010; Volume 470.

20. Zhang, J.; Luo, Y.; Ouyang, B.; Deng, K.; Lu, Z.; Luo, J. Design of an optical reference cavity with low thermal noise limit and flexible thermal expansion properties. Eur. Phys. J. D 2013, 67, 46. [CrossRef]

21. Cole, G.D.; Zhang, W.; Martin, M.J.; Ye, J.; Aspelmeyer, M. Tenfold reduction of Brownian noise in high-reflectivity optical coatings. Nat. Photonics 2013, 67, 46. [CrossRef]

22. Bruckner, F.; Friedrich, D.; Clausnitzer, T.; Britzger, M.; Burmeister, O.; Danzmann, K.; Kley, E.B.; Tunnermann, A.; Schnabel, R. Realization of amonolithic high-reflectivity cavity mirror from a single silicon crystal. Phys. Rev. Lett. 2010, $104,163903$. [CrossRef]

23. Xu, G.; Zhang, L.; Liu, J.; Gao, J.; Chen, L.; Dong, R.; Liu, T.; Zhang, S. Estimation of thermal noise for spindle optical reference cavities. Opt. Commun. 2016, 360, 61-67. [CrossRef]

24. Robinson, J.M.; Oelker, E.; Milner, W.R.; Kedar, D.; Zhang, W.; Legero, T.; Matei, D.G.; Hafner, S.; Riehle, F.; Sterr, U.; et al. Thermal noise and mechanical loss of $\mathrm{SiO}_{2} / \mathrm{Ta}_{2} \mathrm{O}_{5}$ optical coatings at cryogenic temperatures. Opt. Lett. 2021, 46, 592-595. [CrossRef] [PubMed]

25. Webster, S.; Gill, P. Force-insensitive optical cavity. Opt. Lett. 2011, 36, 3572. [CrossRef]

26. Leibrandt, D.R.; Thorpe, M.J.; Notcutt, M.; Drullinger, R.E.; Rosenband, T.; Bergquist, J.C. Spherical reference cavities for frequency stabilization of lasers in non-laboratory environments. Opt. Express 2011, 19, 3471-3482. [CrossRef] [PubMed]

27. Leibrandt, D.R.; Thorpe, M.J.; Bergquist, J.C.; Rosenband, T. Field-test of a robust, portable, frequency-stable laser. Opt. Express 2011, 19, 10278-10286. [CrossRef]

28. Vogt, S.; Lisdat, C.; Legero, T.; Sterr, U.; Ernsting, I.; Nevsky, A.; Schiller, S. Demonstration of a transportable 1 Hz-linewidth laser. Appl. Phys. B 2011, 104, 741. [CrossRef]

29. Argence, B.; Argence, B.; Prevost, E.; Lévèque, T.; Le Goff, R.; Bize, S.; Lemonde, P.; Santarelli, G. Prototype of an ultra-stable optical cavity for space applications. Opt. Express 2012, 20, 25409-25420. [CrossRef]

30. Leibrandt, D.R.; Bergquist, J.C.; Rosenband, T. Cavity-stabilized laser with acceleration sensitivity below $10^{-12} \mathrm{~g}^{-1}$. Phys. Rev. A 2013, 87, 023829. [CrossRef]

31. Chen, Q.; Nevsky, A.; Cardace, M.; Schiller, S.; Legero, T.; Häfner, S.; Uhde, A.; Sterr, U. A compact, robust, and transportable ultra-stable laser with a fractional frequency instability of $1 \times 10^{-15}$. Rev. Sci. Instrum. 2014, 85, 113107. [CrossRef] [PubMed]

32. Świerad, D.; Häfner, S.; Vogt, S.; Venon, B.; Holleville, D.; Bize, S.; Kulosa, A.; Bode, S.; Singh, Y.; Bongs, K.; et al. Ultra-stable clock laser system development towards space applications. Sci. Rep. 2016, 6, 33973. [CrossRef] [PubMed]

33. Koller, S.B.; Grotti, J.; Vogt, S.; Al-Masoudi, A.; Dörscher, S.; Häfner, S.; Sterr, U.; Lisdat, C. Transportable Optical Lattice Clock with $7 \times 10^{-17}$ Uncertainty. Phys. Rev. Lett. 2017, 187, 073601. [CrossRef]

34. Cao, J.; Zhang, P.; Shang, J.; Cui, K.; Yuan, J.; Chao, S.; Wang, S.; Shu, H.; Huang, X. A compact, transportable single-ion optical clock with $7.8 \times 10^{-17}$ systematic uncertainty. Appl. Phys. B 2017, 123, 112. [CrossRef]

35. SOC2-Towards Neutral-Atom Space Optical Clocks. Available online: http://www.exphy.uni-duesseldorf.de/optical_clock/ soc2/index.php (accessed on 5 July 2021).

36. LISA-Laser Interferometer Space Antenna-NASA Home Page. Available online: https://lisa.nasa.gov/index.html (accessed on 5 July 2021).

37. Luo, J.; Sheng, L.; Duan, H.; Gong, Y.; Hu, S.; Ji, J.; Liu, Q.; Mei, J.; Milyukov, V.; Sazhin, M.; et al. TianQin: A space-borne gravitational wave detector. Class. Quantum Grav. 2016, 33, 035010. [CrossRef]

38. Tao, B.; Chen, Q. A vibration-sensitive-cavity design holds impact of higher than 100g. Appl. Phys. B 2018, 124, 228. [CrossRef]

39. Hafiz, M.A.; Ablewski, P.; Masoudi, A.A.; Martínez, H.Á.; Balling, P.; Barwood, G.; Benkler, E.; Bober, M.; Borkowski, M.; Bowden, W.; et al. Guidelines for developing optical clocks with 10-18 fractional frequency uncertainty. arXiv 2019, arXiv:1906.11495. 
40. Chen, X.; Jiang, Y.; Li, B.; Yu, H.; Jiang, H.; Wang, T.; Yao, Y.; Ma, L. Laser frequency instability of $6 \times 10^{-16}$ using $10-\mathrm{cm}-$ long cavities on a cubic spacer. Chin. Opt. Lett. 2020, 18, 030201. [CrossRef]

41. Wang, S.; Cao, J.; Yuan, J.; Liu, D.; Shu, H.; Huang, X. Integrated multiple wavelength stabilization on a multi-channel cavity for a transportable optical clock. Opt. Express 2020, 28, 11852-11860. [CrossRef] [PubMed]

42. Cerdonio, M.; Conti, L.; Heidmann, A.; Pinard, M. Thermoelastic effects at low temperatures and quantum limits in displacement measurements. Phys. Rev. D 2001, 63, 082003. [CrossRef]

43. Jiang, H. Development of Ultra-Stable Laser Sources and Long-Distance Optical Link via Telecommunication Networks. Ph.D. Thesis, University Pairs, Pairs, France, 2010. 